\title{
Numerical method for weights adjustment in minimax multi-model LQ-control
}

\author{
Alexander Poznyak ${ }^{1}$, Francisco Javier Bejarano ${ }^{1, *, \dagger}$ and Leonid Fridman ${ }^{2}$ \\ ${ }^{1}$ CINVESTAV-IPN, Departamento de Control Automático, A.P. 14-740, CP 07000, México, D.F., Mexico \\ ${ }^{2}$ Facultad de Ingenieria, National Autonomous University of Mexico, DEP-FI, UNAM, A.P. 70-256, \\ CP 04510, México, D.F., Mexico
}

\begin{abstract}
SUMMARY
The minimax linear quadratic problem, where ' $m a x$ ' is taken over a finite set of indices (models) and ' $m$ in' is taken over the set of admissible controls, is considered. The solution is obtained by the robust optimal control application. The control turns out to be a linear combination of the controls optimal for each individual model. This paper develops a numerical method for the optimal weights adjustment. An example shows a quick convergence of the proposed procedure. Copyright (C) 2007 John Wiley \& Sons, Ltd.
\end{abstract}

Received 10 May 2006; Revised 16 February 2007; Accepted 27 February 2007

KEY WORDS: minimax control; linear quadratic problem; numerical methods

\section{INTRODUCTION}

Dealing with designing of control for some uncertain systems, there exist situations when the model of the system cannot be defined exactly since the more adequate model can depend on several possible scenarios. In this case, the control can be designed as a multi-model control (see, e.g. [1-4]). To design such control, the minimax approach has been suggested (see [5,6]) where ' $m a x$ ' is taken over all possible models (scenarios) and 'min' is taken over all admissible controls. Evidently that such control does not depend on an individual model and serves simultaneously for the set of possible scenarios. The minimax approach was generalized in [7,8]. Specifically the version of the robust maximum principle for a linear quadratic (LQ) problem was considered in details. The minimax problem was considered in the following aspect. One has $N$ linear state dynamics equations (ODE) each of them corresponding a possible model. The same control law should be applied to all of them in such a way that the worst LQ-index would be minimal over

\footnotetext{
*Correspondence to: Francisco Javier Bejarano, CINVESTAV-IPN, Departamento de Control Automático, A.P. 14-740, CP 07000, México, D.F., Mexico.

${ }^{\dagger}$ E-mail: javier210879@ hotmail.com, javbejarano@yahoo.com.mx
} 
all admissible controls. Such robust optimal control is shown to be a weighted combination of the controls optimal for each individual model. Hence, the problem is reduced to a finite dimensional optimization problem since this robust optimal control depends on a weighting vector belonging to the $N$-dimensional simplex which should be selected providing a minimal value for the original worst LQ-functional. Finding an analytical expression for this function as a function of the weights seems to be very difficult task. In the simplest cases with two $(N=2)$ and three $(N=3)$ models such expression can easily obtained in a graphic form using standard PC. However, for more complex situations $(N \geqslant 4)$ such approach cannot be realized and any numerical procedure for the corresponding weight adjustment (optimization) is welcomed.

In [9-11] was considered a similar (to the first glance) problem. But, in fact, it differs in many aspects with the problem which we are dealing with. First, the dynamics, considered in the references above, does not contain any exogenous input. Second, they considered only a single plant with a multi-criterion cost functional. In our paper, we are dealing with a really multi-plant (or multi-model dynamic system) but having the same criterion for each possible scenario. Evidently, these two problems have a different philosophical treatment, but, sure, they may be attacked by closed mathematical methods. The main difference between these two considerations consists in the corresponding differential Riccati equations used in the min-max feedback realization: we deal with different matrices $A_{i}$ and $B_{i}$ in the differential equation, governing the considered dynamics, but with the same matrix $R$ (the control-cost matrix); in the papers, cited above, the authors have only a single matrix $A$ and a single matrix $B$ but several $R_{i}$. The corresponding weight dependence in both cases is absolutely different since we have the right-hand side of the Riccati ODE which is linearly dependent on weights, and in the cited papers this dependence is essentially nonlinear that significantly complicates the analysis of the weight-adjustment procedure. Moreover, there is no any convergence analysis of the suggested iterative weight-adjustment procedure in the papers referred above (only [11] contains some brief scheme of the convergence analysis, but not a proof).

In this paper, we suggest a new numerical (iterative) method which provides a quick convergence of the weighting vector to its optimal value. This numerical method practically makes workable the realization of the robust optimal control suggested in $[7,8]$ and complements the results given in $[12-14]$.

\subsection{Structure of the paper}

In Section 2, the model description is presented and the purpose of the control law is formulated. A preliminary result, needed for the following consideration, is given in Section 3. Then the iterative numerical procedure is suggested. The convergence analysis of this numerical procedure is given in Section 4. An example, illustrating a quick convergence of the method, is presented in Section 5 .

\section{MOTIVATION AND PROBLEM STATEMENT}

Let us consider a set of linear state models given by

$$
\dot{x}^{\alpha}(t)=A^{\alpha}(t) x^{\alpha}(t)+B^{\alpha}(t) u(t)+d^{\alpha}(t), \quad x^{\alpha}(0)=x_{0}^{\alpha}
$$

where the index $\alpha$ belongs to a finite set, that is, $\alpha \in \overline{1, N}$, (N is a positive integer), $x^{\alpha}(t), d^{\alpha}(t) \in$ $R^{n}, u(t) \in \mathbb{R}^{m}$ and $A^{\alpha}(t), B^{\alpha}(t), d^{\alpha}(t)$ are continuous functions on $t \in[0, T]$. Let us define the 
performance index as

$$
h^{\alpha}:=\frac{1}{2}\left(x^{\alpha}(T), G^{\alpha} x^{\alpha}(T)\right)+\frac{1}{2} \int_{t=0}^{\mathrm{T}}\left[\left(x^{\alpha}(t), Q^{\alpha} x^{\alpha}(t)\right)+(u(t), R u(t))\right] \mathrm{d} t
$$

where $Q^{\alpha} \geqslant 0, G^{\alpha} \geqslant 0, R>0$. The minimax LQ control problem was formulated in [8] as

$$
\max _{\alpha \in \overline{1, N}} h^{\alpha} \longrightarrow \min _{u \in \mathbb{R}^{m}}
$$

The solution of this problem, also given in [8], is as follows. Define the following extended system:

$$
\dot{\mathbf{x}}(t)=\mathbf{A x}(t)+\mathbf{B} u(\mathbf{x}, t)+\mathbf{d}
$$

where

$$
\begin{gathered}
\mathbf{x}:=\left[\begin{array}{c}
x^{1}(t) \\
\vdots \\
x^{N}(t)
\end{array}\right], \quad \mathbf{A}:=\left[\begin{array}{ccc}
A^{1}(t) & 0 \cdots & 0 \\
0 & & \vdots \\
\vdots & \ddots & 0 \\
0 & \cdots 0 & A^{N}(t)
\end{array}\right], \quad \mathbf{B}:=\left[\begin{array}{c}
B^{1}(t) \\
\vdots \\
B^{N}(t)
\end{array}\right], \quad \mathbf{d}:=\left[\begin{array}{c}
d^{1}(t) \\
\vdots \\
d^{N}(t)
\end{array}\right] \\
\mathbf{Q}:=\left[\begin{array}{ccc}
Q_{1} & 0 \cdots & 0 \\
0 & \ddots & \vdots \\
\vdots & & 0 \\
0 & \cdots 0 & Q_{N}
\end{array}\right], \quad \mathbf{G}:=\left[\begin{array}{ccc}
G_{1} & 0 \cdots & 0 \\
0 & & \vdots \\
\vdots & \ddots & 0 \\
0 & \cdots 0 & G_{N}
\end{array}\right], \quad \boldsymbol{\Lambda}:=\left[\begin{array}{ccc}
\lambda_{1} I_{n \times n} & 0 \cdots & 0 \\
0 & & \vdots \\
\vdots & & 0 \\
0 & \cdots 0 & \lambda_{N} I_{n \times n}
\end{array}\right]
\end{gathered}
$$

with $\lambda=\left(\lambda_{1}, \ldots, \lambda_{N}\right) \in S^{N}$

$$
S^{N}=\left\{\lambda \in \mathbb{R}^{N}: \lambda_{i} \geqslant 0, \sum_{i=1}^{N} \lambda_{i}=1\right\}
$$

Then, the robust optimal control realizing (3) is of the form

$$
u=-R^{-1} \mathbf{B}^{\mathrm{T}}\left(\mathbf{P}_{\lambda} \mathbf{x}+\mathbf{p}_{\lambda}\right)
$$

where the matrix $\mathbf{P}_{\lambda}=\mathbf{P}_{\lambda}^{\mathrm{T}} \in R^{n N \times n N}$ is the solution of the parameterized differential matrix Riccati equation:

$$
\dot{\mathbf{P}}_{\lambda}+\mathbf{P}_{\lambda} \mathbf{A}+\mathbf{A}^{\mathrm{T}} \mathbf{P}_{\lambda}-\mathbf{P}_{\lambda} \mathbf{B} R^{-1} \mathbf{B}^{\mathrm{T}} \mathbf{P}_{\lambda}+\Lambda \mathbf{Q}=0, \quad \mathbf{P}_{\lambda}(T)=\mathbf{\Lambda} \mathbf{G}
$$

and the shifting vector $\mathbf{p}_{\lambda}$ satisfies

$$
\dot{\mathbf{p}}_{\lambda}+\mathbf{A}^{\mathrm{T}} \mathbf{p}_{\lambda}-\mathbf{P}_{\lambda} \mathbf{B} R^{-1} \mathbf{B}^{\mathrm{T}} \mathbf{p}_{\lambda}+\mathbf{P}_{\lambda} \mathbf{d}=\mathbf{0}, \quad \mathbf{p}_{\lambda}(T)=0
$$


Thus, the solution of (3) is reduced to the finding of the optimal weighting vector $\lambda^{*}$ which solves the following finite dimensional optimization problem:

$$
\begin{aligned}
\lambda^{*}= & \arg \min _{\lambda \in S^{N}} J(\lambda) \\
J(\lambda):= & \max _{\alpha=1, N} h^{\alpha} \\
= & \frac{1}{2} \mathbf{x}^{\mathrm{T}}(0) \mathbf{P}_{\lambda}(0) \mathbf{x}(0)+\mathbf{x}^{\mathrm{T}}(0) \mathbf{p}_{\lambda}(0) \\
& +\frac{1}{2} \max _{\alpha=\overline{1, N}}\left[x^{\alpha \mathrm{T}}(T) G^{\alpha} x^{\alpha}(T)+\int_{t=0}^{\mathrm{T}} x^{\alpha \mathrm{T}}(t) Q^{\alpha} x^{\alpha}(t) \mathrm{d} t\right] \\
& -\frac{1}{2} \sum_{\alpha=1}^{N} \lambda_{i}\left[x^{\alpha \mathrm{T}}(T) G^{\alpha} x^{\alpha}(T)+\int_{t=0}^{\mathrm{T}} x^{\alpha \mathrm{T}}(t) Q^{\alpha} x^{\alpha}(t) \mathrm{d} t\right] \\
& +\frac{1}{2} \int_{t=0}^{\mathrm{T}} \mathbf{p}_{\lambda}^{\mathrm{T}}\left[2 \mathbf{d}-\mathbf{B} R^{-1} \mathbf{B}^{\mathrm{T}} \mathbf{p}_{\lambda}\right] \mathrm{d} t
\end{aligned}
$$

Thus, the goal of this paper is to develop a numerical method which allows to find the optimal weighting vector $\lambda^{*}$ for any finite number $N$ of possible models (or, scenarios).

\section{PRELIMINARY RESULTS}

\section{Lemma 1}

Let $\lambda^{*}$ be a minimum point, that is, $J\left(\lambda^{*}\right) \leqslant J(\lambda)$ for all $\lambda \in S^{N}$. Then, for any active index $\alpha \in \overline{1, N}$ such that $1 \geqslant \lambda_{\alpha}^{*}>0$, the functional $h^{\alpha}\left(\lambda^{*}\right)$ satisfies the following equality:

$$
h^{\alpha}\left(\lambda^{*}\right)=J\left(\lambda^{*}\right)
$$

and for all inactive indices $\alpha$ such that $\lambda_{\alpha}^{*}=0$

$$
h^{\alpha}\left(\lambda^{*}\right) \leqslant J\left(\lambda^{*}\right)
$$

Proof

If for some $j \in \overline{1, N}$ we have $h^{j}\left(\lambda^{*}\right)>J\left(\lambda^{*}\right)$, then

$$
J\left(\lambda^{*}\right)=\max _{\alpha \in \overline{1, N}} h^{\alpha}\left(\lambda^{*}\right) \geqslant h^{j}\left(\lambda^{*}\right)>J\left(\lambda^{*}\right)
$$

that leads to a contradiction. So, for all indices $\alpha$ it follows $h^{\alpha}\left(\lambda^{*}\right) \leqslant J\left(\lambda^{*}\right)$. Result (9) for active indices follows directly from the complementary slackness condition established in [8]. 
Corollary 1

The optimal performance index $J\left(\lambda^{*}\right)$ can be represented as

$$
J\left(\lambda^{*}\right)=\frac{1}{2} \mathbf{x}^{\mathrm{T}}(0) \mathbf{P}_{\lambda^{*}}(0) \mathbf{x}(0)+\mathbf{x}^{\mathrm{T}}(0) \mathbf{p}_{\lambda^{*}}(0)+\frac{1}{2} \int_{t=0}^{\mathrm{T}} \mathbf{p}_{\lambda^{*}}^{\mathrm{T}}\left[2 \mathbf{d}-\mathbf{B} R^{-1} \mathbf{B}^{\mathrm{T}} \mathbf{p}_{\lambda^{*}}\right] \mathrm{d} t
$$

Proof

Adding and subtracting the integral of $u^{\mathrm{T}}(t) R u(t)$ in (8), we get

$$
\begin{aligned}
J(\lambda)= & \frac{1}{2} \mathbf{x}^{\mathrm{T}}(0) \mathbf{P}_{\lambda}(0) \mathbf{x}(0)+\mathbf{x}^{\mathrm{T}}(0) \mathbf{p}_{\lambda}(0) \\
& +\left[J(\lambda)-\sum_{i=1}^{N} \lambda_{\alpha} h^{\alpha}(\lambda)\right]+\frac{1}{2} \int_{t=0}^{\mathrm{T}} \mathbf{p}_{\lambda}^{\mathrm{T}}\left[2 \mathbf{d}-\mathbf{B} R^{-1} \mathbf{B}^{\mathrm{T}} \mathbf{p}_{\lambda}\right] \mathrm{d} t
\end{aligned}
$$

Therefore, taking $\lambda=\lambda^{*}$, in view of Lemma 1 , specifically (9), and since $\sum_{\alpha=1}^{N} \lambda_{\alpha}=1$, we find that $J\left(\lambda^{*}\right)=\sum_{i=1}^{N} \lambda_{\alpha}^{*} h^{\alpha}\left(\lambda^{*}\right)$. Hence the performance index $J\left(\lambda^{*}\right)$ is as in (11).

Corollary 2

If the vector $\lambda^{*}$ is a minimum point, then for any $\gamma>0$

$$
\lambda^{*}=\pi\left\{\lambda^{*}+\gamma F\left(\lambda^{*}\right)\right\}
$$

where $\pi\{\cdot\}$ is the projector to the simplex $S^{N}$, that is,

$$
\|\pi\{x\}-x\|<\|\lambda-x\| \quad \text { for any } \lambda \in S^{N}, \quad \lambda \neq \pi\{x\}
$$

and $F(\lambda) \in \mathbb{R}^{N}$ is the vector whose $i$ th term is the performance functional $h^{i}$, i.e.

$$
F(\lambda)=\left[\begin{array}{c}
h^{1}(\lambda) \\
\vdots \\
h^{N}(\lambda)
\end{array}\right]
$$

Proof

Since $S^{N}$ is a closed convex set, the following property holds:

$$
\text { for any } x \in \mathbb{R}^{n}, \quad \mu=\pi\{x\} \Longleftrightarrow(x-\mu, \lambda-\mu) \leqslant 0 \quad \text { for all } \lambda \in S^{N}
$$

Let $\lambda_{i_{j}}^{*}, j=\overline{1, r}$ be the components of $\lambda^{*}$ different from zero and $\lambda_{i_{k}}^{*} k=\overline{r+1, N}$ the components of $\lambda^{*}$ equal to zero. Thus, taking into account Lemma 1 and, since $\lambda_{i_{k}}-\lambda_{i_{k}}^{*} \geqslant 0\left(\lambda_{i_{k}}^{*}=0\right)$, we obtain

$$
\begin{aligned}
& \left(\lambda^{*}+\gamma F\left(\lambda^{*}\right)-\lambda^{*}, \lambda-\lambda^{*}\right) \\
& \quad=\gamma\left[J\left(\lambda^{*}\right) \sum_{j=1}^{r}\left(\lambda_{i_{j}}-\lambda_{i_{j}}^{*}\right)+\sum_{k=r+1}^{N} h^{i_{k}}\left(\lambda^{*}\right)\left(\lambda_{i_{k}}-\lambda_{i_{k}}^{*}\right)\right]
\end{aligned}
$$




$$
\leqslant \gamma J\left(\lambda^{*}\right)\left[\sum_{j=1}^{r}\left(\lambda_{i_{j}}-\lambda_{i_{j}}^{*}\right)+\sum_{k=r+1}^{N}\left(\lambda_{i_{k}}-\lambda_{i_{k}}^{*}\right)\right]=\gamma J\left(\lambda^{*}\right) \sum_{j=1}^{N}\left(\lambda_{i_{j}}-\lambda_{i_{j}}^{*}\right)=0
$$

for all $\lambda \in S^{N}$. Thus, by (13), (14) implies $\lambda^{*}=\pi\left\{\lambda^{*}+\gamma F\left(\lambda^{*}\right)\right\}$.

\section{NUMERICAL PROCEDURE FOR THE WEIGHTS ADJUSTMENT}

In [8], there was shown that the control $u(\mathbf{x}, t)$ designed as in (3) is the combination (where the weights are the components $\lambda_{\alpha}$ ) of the controls optimal for each individual model. Hence, it seems to be clear that a bigger weight $\lambda_{\alpha}$ of the control, optimizing the $\alpha$-model, implies a better (smaller) performance index $h^{\alpha}(\lambda)$. This fact may be expressed in the following manner: if $\lambda_{\alpha}^{\prime} \neq \lambda_{\alpha}^{\prime \prime}$

$$
\left(\lambda_{\alpha}^{\prime}-\lambda_{\alpha}^{\prime \prime}\right)\left[h^{\alpha}\left(\lambda^{\prime}\right)-h^{\alpha}\left(\lambda^{\prime \prime}\right)\right]<0
$$

for any $\lambda^{\prime} \neq \lambda^{\prime \prime} \in S^{N}$. Summing (15) on $\alpha \in \overline{1, N}$ leads to the following condition which we will accept as the assumption.

\section{Assumption 1}

For any $\lambda^{\prime} \neq \lambda^{\prime \prime} \in S^{N}$, the following inequality holds:

$$
\left(\lambda^{\prime}-\lambda^{\prime \prime}, F\left(\lambda^{\prime}\right)-F\left(\lambda^{\prime \prime}\right)\right)<0
$$

and the identity in (16) is possible if and only if $\lambda^{\prime}=\lambda^{\prime \prime}$.

\section{Proposition 1}

Under Assumption 1, the functional $J(\lambda)$ has a unique minimum point $\lambda^{*}$.

Proof

We will show that if $\tilde{\lambda}$ differs with $\lambda^{*}$, then $\tilde{\lambda}$ does not satisfy identity (12). If $\tilde{\lambda} \neq \lambda^{*}$, then (14) implies

$$
\begin{aligned}
& \left(\tilde{\lambda}+\gamma F(\tilde{\lambda})-\tilde{\lambda}, \lambda^{*}-\tilde{\lambda}\right) \\
& \quad \geqslant \gamma\left[\left(F(\tilde{\lambda}), \lambda^{*}-\tilde{\lambda}\right)+\left(F\left(\lambda^{*}\right), \tilde{\lambda}-\lambda^{*}\right)\right]=\gamma\left(F(\tilde{\lambda})-F\left(\lambda^{*}\right), \lambda^{*}-\tilde{\lambda}\right)
\end{aligned}
$$

On the other hand, Assumption 1 yields

$$
\gamma\left(F(\tilde{\lambda})-F\left(\lambda^{*}\right), \lambda^{*}-\tilde{\lambda}\right)=-\gamma\left(\tilde{\lambda}-\lambda^{*}, F(\tilde{\lambda})-F\left(\lambda^{*}\right)\right)>0
$$

Thus, both (17) and (18) imply

$$
\left(\tilde{\lambda}+\gamma F(\tilde{\lambda})-\tilde{\lambda}, \lambda^{*}-\tilde{\lambda}\right)>0
$$

But (19) means that $\tilde{\lambda} \neq \pi\{\tilde{\lambda}+\gamma F(\tilde{\lambda})\}$ (see (13)). Therefore, by Corollary 2, we deduce that $\tilde{\lambda}$ is not a minimum point.

Now, we are ready to present a numerical method for the adjustment of the weight vector $\lambda$. 


\subsection{Numerical method}

Define the sequence of vectors $\left\{\lambda^{k}\right\}$ as

$$
\begin{aligned}
\lambda^{k+1} & =\pi\left\{\lambda^{k}+\frac{\gamma^{k}}{J\left(\lambda^{k}\right)+\varepsilon} F\left(\lambda^{k}\right)\right\}, \quad \lambda^{0} \in S^{N}, \quad k=0,1,2, \ldots \\
F\left(\lambda^{k}\right) & =\left[h^{1}\left(\lambda^{k}\right) \cdots h^{N}\left(\lambda^{k}\right)\right]^{\mathrm{T}} \\
J\left(\lambda^{k}\right) & :=\max _{\alpha \in \overline{1, N}} h^{\alpha}\left(\lambda^{k}\right)
\end{aligned}
$$

where $\varepsilon$ is an arbitrary strictly positive (small enough) constant.

\section{Theorem 1}

Let $\lambda^{*}$ be the minimum point for $J(\lambda)$. If

(1) the sequence $\left\{\lambda^{k}\right\}$ is generated by (20);

(2) Assumption 1 holds;

(3) there exists a constant $L$ such that for all $\alpha \in \overline{1, N}$ and for any $\mu, \lambda \in S^{N}$

$$
\left|h^{\alpha}(\mu)-h^{\alpha}(\lambda)\right| \leqslant J(\lambda) L|\mu-\lambda|
$$

(4) the gain sequence $\left\{\gamma^{k}\right\}$ satisfies

$$
\gamma^{k}>0, \quad \sum_{k=0}^{\infty} \gamma^{k}=\infty, \quad \sum_{k=0}^{\infty}\left(\gamma^{k}\right)^{2}<\infty
$$

then

$$
\lim _{k \rightarrow \infty} \lambda^{k}=\lambda^{*}
$$

\section{Proof}

For $v^{k}:=\lambda^{k}-\lambda^{*}$, in view of (12) and the property of projection $\|\pi\{x\}-\pi\{y\}\| \leqslant\|x-y\|$ for all $x, y \in \mathbb{R}^{N}$, we have

$$
\begin{aligned}
\left\|v^{k+1}\right\|^{2} & =\left\|\pi\left\{\lambda^{k}+\frac{\gamma^{k}}{J\left(\lambda^{k}\right)+\varepsilon} F\left(\lambda^{k}\right)\right\}-\lambda^{*}\right\|^{2} \\
& =\left\|\pi\left\{\lambda^{k}+\frac{\gamma^{k}}{J\left(\lambda^{k}\right)+\varepsilon} F\left(\lambda^{k}\right)\right\}-\pi\left\{\lambda^{*}+\frac{\gamma^{k}}{J\left(\lambda^{k}\right)+\varepsilon} F\left(\lambda^{*}\right)\right\}\right\|^{2} \\
& \leqslant\left\|v^{k}+\frac{\gamma^{k}}{J\left(\lambda^{k}\right)+\varepsilon}\left[F\left(\lambda^{k}\right)-F\left(\lambda^{*}\right)\right]\right\|^{2} \\
& =\left\|v^{k}\right\|^{2}+\frac{\left(\gamma^{k}\right)^{2}}{J^{2}\left(\lambda^{k}\right)+2 J\left(\lambda^{k}\right) \varepsilon+\varepsilon^{2}}\left\|F\left(\lambda^{k}\right)-F\left(\lambda^{*}\right)\right\|^{2}
\end{aligned}
$$




$$
\begin{aligned}
& +2 \frac{\gamma^{k}}{J\left(\lambda^{k}\right)+\varepsilon}\left(v^{k}, F\left(\lambda^{k}\right)-F\left(\lambda^{*}\right)\right) \\
\leqslant & \left\|v^{k}\right\|^{2}\left(1+\left(\gamma^{k}\right)^{2} L^{2}\right)+2 \frac{\gamma^{k}}{J\left(\lambda^{k}\right)+\varepsilon}\left(v^{k}, F\left(\lambda^{k}\right)-F\left(\lambda^{*}\right)\right) \\
\leqslant & \left\|v^{k}\right\|^{2}\left(1+\left(\gamma^{k}\right)^{2} L^{2}\right)
\end{aligned}
$$

In the last inequality of (22) we have used Assumption 1. Define the new variable $w^{k}$ by

$$
w^{k}:=\left\|v^{k}\right\|^{2} \prod_{s=k}^{\infty}\left[1+\left(\gamma^{s}\right)^{2} L^{2}\right]
$$

So, (22) implies

$$
\begin{aligned}
w^{k+1} & :=\left\|v^{k+1}\right\|^{2} \prod_{s=k+1}^{\infty}\left[1+\left(\gamma^{s}\right)^{2} L^{2}\right] \\
& \leqslant\left\|v^{k}\right\|^{2}\left(1+\left(\gamma^{k}\right)^{2} L^{2}\right) \prod_{s=k+1}^{\infty}\left[1+\left(\gamma^{s}\right)^{2} L^{2}\right]=w^{k}
\end{aligned}
$$

which means (by the Weierstrass theorem) that the sequence $\left\{w^{k}\right\}$ converges and, hence, there exists the limit

$$
w:=\lim _{k \rightarrow \infty} w^{k}=\lim _{k \rightarrow \infty}\left\|v^{k}\right\|^{2}
$$

But, from (22), we have also the inequality

$$
\begin{aligned}
2 \frac{\gamma^{k}}{J\left(\lambda^{k}\right)+\varepsilon}\left|\left(v^{k}, F\left(\lambda^{k}\right)-F\left(\lambda^{*}\right)\right)\right| & \leqslant\left\|v^{k}\right\|^{2}\left(1+\left(\gamma^{k}\right)^{2} L^{2}\right)-\left\|v^{k+1}\right\|^{2} \\
& =\frac{w^{k}-w^{k+1}}{\prod_{s=k+1}^{\infty}\left[1+\left(\gamma^{s}\right)^{2} L^{2}\right]} \leqslant w^{k}-w^{k+1}
\end{aligned}
$$

Summation it by $k$ from 0 up to $\infty$ yields

$$
2 \sum_{k=0}^{\infty} \gamma^{k} \frac{\left|\left(v^{k}, F\left(\lambda^{k}\right)-F\left(\lambda^{*}\right)\right)\right|}{J\left(\lambda^{k}\right)+\varepsilon} \leqslant w^{0}-w<\infty
$$

In view of the property $\sum_{k=0}^{\infty} \gamma^{k}=\infty$, it follows that there exists a subsequence $k_{t}(t=1,2, \ldots)$ such that

$$
\frac{\left|\left(v^{k_{t}}, F\left(\lambda^{k_{t}}\right)-F\left(\lambda^{*}\right)\right)\right|}{J\left(\lambda^{k_{t}}\right)+\varepsilon} \underset{t \rightarrow \infty}{\longrightarrow} 0
$$

Since $J\left(\lambda^{k_{t}}\right)$ is bounded, then, by (16), this implies $\lambda^{k_{t}} \underset{t \rightarrow \infty}{\longrightarrow} \lambda^{*}$, or, equivalently,

$$
\lim _{t \rightarrow \infty} w^{k_{t}}=\lim _{t \rightarrow \infty}\left\|v^{k_{t}}\right\|^{2}=0
$$


But $\left\{w^{k}\right\}$ converges to $w$. So all its subsequences converges to the same limit that implies $w=0$. Theorem is proven.

\section{EXAMPLE}

The following example illustrates the proposed numerical method (20) in the case $N=3$ where the parameters of possible models are as follows:

$$
\begin{aligned}
& A_{1}=\left[\begin{array}{ccc}
-2 & 0.5 & 1 \\
0.5 & 1.2 & -2 \\
1 & 2 & -1.5
\end{array}\right], \quad A_{2}=\left[\begin{array}{ccc}
-0.3 & 1.5 & -0.15 \\
-1 & 0.12 & 2 \\
1 & 2 & -3
\end{array}\right], \quad A_{3}=\left[\begin{array}{ccc}
0.4 & -1 & 0.3 \\
0.5 & -0.4 & 0.3 \\
0.5 & 0.6 & -1
\end{array}\right] \\
& B_{1}=\left[\begin{array}{c}
0.5 \\
1 \\
1
\end{array}\right], \quad B_{2}=\left[\begin{array}{c}
1.5 \\
-2 \\
1
\end{array}\right], \quad B_{3}=\left[\begin{array}{c}
0.5 \\
0.2 \\
1
\end{array}\right] \\
& d^{1}=\left[\begin{array}{c}
0.1 \\
0.05 \\
0.01
\end{array}\right], \quad d^{2}=\left[\begin{array}{c}
0.1 \sin (t) \\
0.2 \sin (t / 2) \\
0.1
\end{array}\right], \quad d^{3}=\left[\begin{array}{c}
0.1 \\
0.05 \cos (t) \\
0.1
\end{array}\right]
\end{aligned}
$$

Table I. Values of $\lambda^{k}$ and $h^{\alpha}\left(\lambda^{k}\right)$.

\begin{tabular}{cccccccc}
\hline$k$ & $\lambda_{1}$ & $\lambda_{2}$ & $\lambda_{3}$ & $h^{1}$ & $h^{2}$ & $h^{3}$ & $J$ \\
\hline 1 & 0.5 & 0.4 & 0.1 & 116.060 & 109.897 & 977.037 & 977.037 \\
2 & 0.208365 & 0.102057 & 0.689576 & 236.303 & 1116.18 & 310.533 & 1116.18 \\
3 & 0.065899 & 0.353738 & 0.580362 & 489.798 & 349.164 & 503.783 & 503.783 \\
4 & 0.093832 & 0.288619 & 0.617548 & 360.415 & 462.742 & 460.030 & 462.742 \\
5 & 0.057465 & 0.307535 & 0.634999 & 555.476 & 432.953 & 451.644 & 555.476 \\
6 & 0.084632 & 0.290587 & 0.624780 & 393.174 & 461.878 & 455.737 & 461.878 \\
7 & 0.068843 & 0.299589 & 0.631567 & 471.404 & 447.301 & 453.104 & 471.404 \\
8 & 0.073125 & 0.296569 & 0.630305 & 446.649 & 452.593 & 453.386 & 453.386 \\
9 & 0.071960 & 0.297042 & 0.630997 & 453.083 & 451.886 & 452.979 & 453.083 \\
10 & 0.072066 & 0.296855 & 0.631077 & 452.488 & 452.251 & 452.875 & 452.875 \\
$\vdots$ & $\vdots$ & $\vdots$ & $\vdots$ & $\vdots$ & $\vdots$ & $\vdots$ & $\vdots$ \\
31 & 0.072036 & 0.296664 & 0.631299 & 452.660 & 452.656 & 452.665 & 452.665 \\
32 & 0.072036 & 0.296664 & 0.631299 & 452.661 & 452.657 & 452.664 & 452.664 \\
33 & 0.072036 & 0.296663 & 0.631300 & 452.666 & 452.658 & 452.664 & 452.665 \\
34 & 0.072036 & 0.296663 & 0.631300 & 452.661 & 452.658 & 452.664 & 452.664 \\
35 & 0.072036 & 0.296663 & 0.631300 & 452.660 & 452.658 & 452.664 & 452.664 \\
36 & 0.072035 & 0.296663 & 0.631300 & 452.660 & 452.660 & 452.663 & 452.663 \\
37 & 0.072035 & 0.296663 & 0.631300 & 452.661 & 452.659 & 452.663 & 452.663 \\
38 & 0.072035 & 0.296663 & 0.631300 & 452.660 & 452.660 & 452.663 & 452.663 \\
39 & 0.072035 & 0.296663 & 0.631301 & 452.662 & 452.659 & 452.663 & 452.663 \\
40 & 0.072035 & 0.296663 & 0.631301 & 452.662 & 452.659 & 452.663 & 452.663 \\
\hline
\end{tabular}




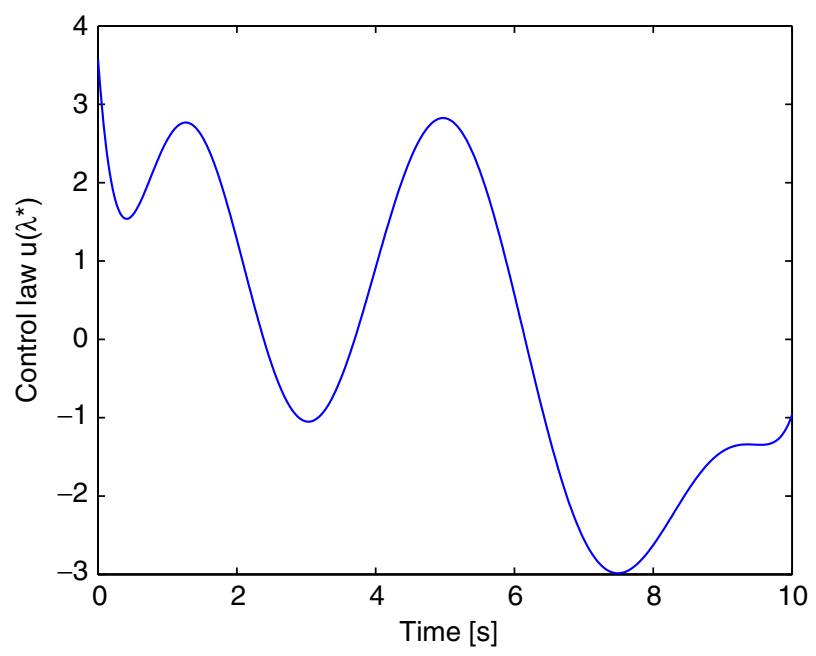

Figure 1. Control law $u$ for $\lambda^{*}$.

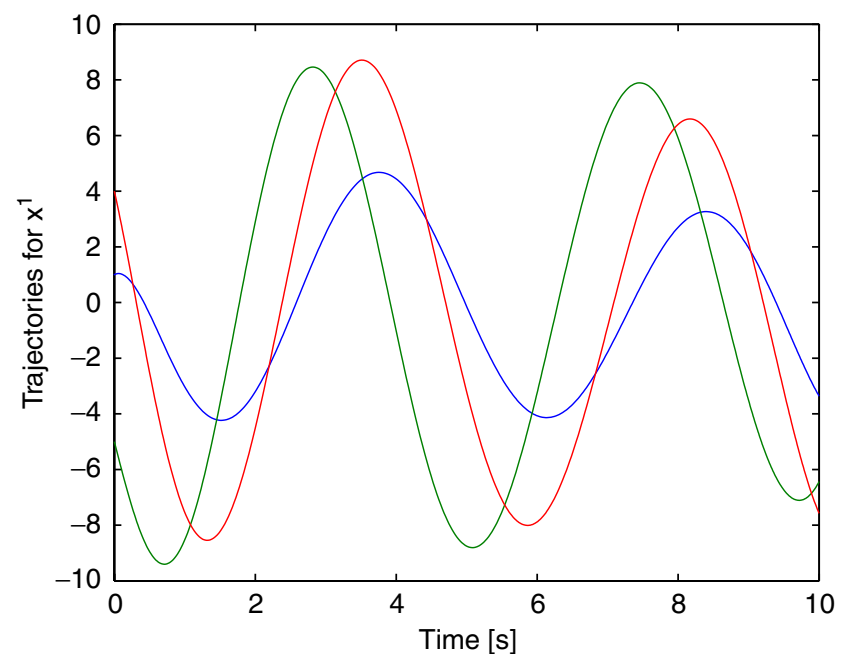

Figure 2. Trajectories of the state corresponding to $\alpha=1$.

We select the matrices $Q^{\alpha}=G^{\alpha}=I, R=1$. Using the gain-step sequence $\left\{\gamma^{k}\right\}$ (20) with $\gamma^{k}=1 /(k+1), k=0,1,2, \ldots$, we obtained the results presented in Table I. There are shown the values of the vector $\lambda^{k}$ and the performance index $h^{\alpha}\left(\lambda^{k}\right)$ for each iteration $k$.

From Table I, one can see that the weights practically converge after 10 iterations. Since all indices are active $\left(\lambda_{\alpha}^{*}>0\right)$, all performance functional $h^{\alpha}\left(\lambda^{k}\right)$ practically turn out to be equal after 


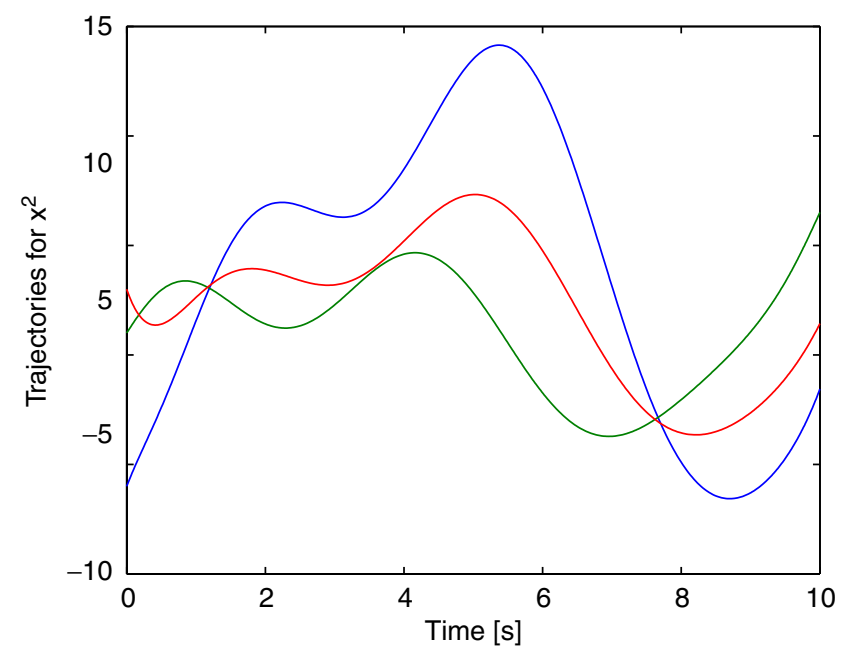

Figure 3. Trajectories of the state corresponding to $\alpha=2$.

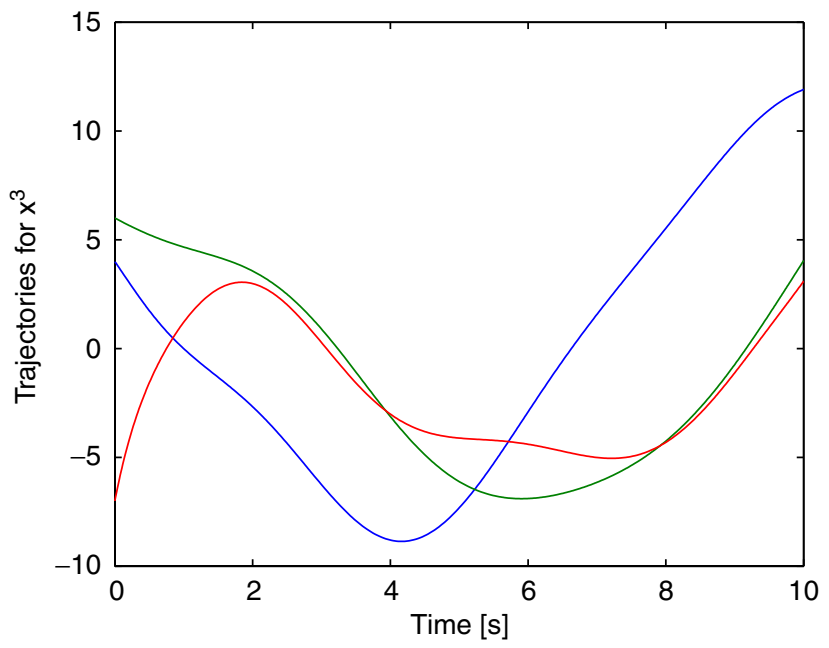

Figure 4. Trajectories of the state corresponding to $\alpha=3$.

40 iterations. Thus, we have $\lambda^{*} \approx(0.072035,0.296663,0.631301)$. The control law $u=u\left(\lambda^{*}\right)$ is depicted in Figure 1. Figures 2-4 show the trajectories of $x^{\alpha}$ for $\alpha=1,2,3$.

\section{CONCLUSIONS}

In this paper, the numerical method for finding the optimal weights in the robust optimal control representation is proposed. The suggested procedure is workable without any changes in the 
gain-step sequence and shows a quick convergence. It may be successfully applied also for multimodel stochastic LQ-control [15].

\section{REFERENCES}

1. Ackermann J, Kaesbaver D, Sienel W, Steinhauser R. Robust Control Systems with Uncertain Physical Parameters. Springer: Berlin, 1994.

2. Murray-Smith R, Johansen TA. Multiple Model Approaches to Modeling and Control. Taylor \& Francis: London, 1997.

3. Ksouri-Lahmari M, Kamel AE, Benrejeb M, Borne P. Multimodel, multicontrol decision making in system automation. IEEE-SMC'97, Orlando, Florida, U.S.A., October 1997.

4. Magni J-F, Gorrec Y, Chiappa C. An observer based multimodel control design approach. International Journal of Systems Science 1999; 30(1):61-68.

5. Demyanov VF, Malozemov V. Introduction to Minimax. Dover: New York, 1990.

6. Schmitendorf W. A sufficient condition for minmax control of systems with uncertainty in the state equations. IEEE Transactions on Automatic Control 1976; 21(4):512-515.

7. Boltyansky V, Poznyak A. Robust maximum principle in minimax control. International Journal of Control 1999; 72(4):305-314.

8. Poznyak A, Duncan T, Pasik-Duncan B, Boltyansky V. Robust maximum principle for minimax linear quadratic problem. International Journal of Control 2002; 75(15):1170-1177.

9. Li D. On the minimax solution of multiple linear-quadratic problems. IEEE Transaction on Automatic Control 1990; 35(10):1153-1156.

10. Carvalho J, Ferreira P. Multiple-criterion control: a convex programming approach. Automatica 1995; 31(7): $1025-1029$.

11. Ng W-L, Lai K-K. An efficient scheme for minimax solutions of multiple linear-quadratic control. Optimal Control Applications and Methods 2005; 26(6):337-344.

12. Poznyak A, Fridman L, Bejarano F. Mini-max integral sliding mode control for multimodel linear uncertain systems. IEEE Transactions on Automatic Control 2004; 49(1):97-102.

13. Poznyak AS, Shtessel YB, Gallegos CJ. Mini-max sliding mode control for multi-model linear time varying systems. IEEE Transactions on Automatic Control 2003; 48(12):2141-2150.

14. Fridman L, Poznyak A, Bejarano F. Decomposition of the min-max multimodel problem via integral sliding mode. International Journal of Robust and Nonlinear Control 2005; 15(13):559-574.

15. Poznyak A, Duncan T, Pasik-Duncan B, Boltyansky V. Robust optimal control for minimax stochastic linear quadratic problem. International Journal of Control 2002; 75(14):1054-1065. 